\title{
DEVELOPMENT OF 230 GHZ RADIO RECEIVER SYSTEM FOR SRAO
}

\author{
Jung-Won Lee ${ }^{1}$, Chang-Hee Kim² ${ }^{2}$, Hyunwoo Kang ${ }^{1}$, Bangwon LeE ${ }^{2}$, Junghwan Han ${ }^{2}$, SeOk-Ho LeE ${ }^{2}$, \\ Il-Gyo JEONG ${ }^{2}$, BON-Chul KoO ${ }^{2}$, AND Yong-Sun PARK ${ }^{2}$ \\ ${ }^{1}$ Korea Astronomy and Space Science Institute, Daejeon 305-348, Korea \\ E-mail : jwl@kasi.re.kr \\ 2 Department of Physics and Astronomy, Seoul National University, Seoul 151-742, Korea \\ (Received September 17, 2013; Accepted November 4, 2013)
}

\begin{abstract}
We develop a radio receiver system operating at $\lambda \sim 1.3 \mathrm{~mm}$ for the $6 \mathrm{~m}$ telescope of Seoul Radio Astronomy Observatory. It consists of a dual polarization receiver, a couple of IF processing units, two FFT spectrometers, and associated software. By adopting sideband-separating superconductor mixers with image band terminated to waveguide load at $4.2 \mathrm{~K}$, we achieve $T_{\mathrm{RX}} \leq 100 \mathrm{~K}$ and $T_{\text {sys }}$ less than 150 $\mathrm{K}$ at best weather condition over $210-250 \mathrm{GHz}$ frequency range. The intermediate frequency signal of 3.5-4.5 GHz is down converted to 0-1 GHz and fed into the FFT spectrometers. The spectrometer covers $1 \mathrm{GHz}$ bandwidth with a spectral resolution of $61 \mathrm{KHz}$. Test observations are conducted toward several radio sources to evaluate the performance of the system. Aperture and beam efficiencies measured by observing planets are found to be typically $44 \sim 59 \%$ and $47 \sim 61 \%$, respectively over the $\mathrm{RF}$ band, which are consistent with those measured at $3 \mathrm{~mm}$ band previously.
\end{abstract}

Key words : instrumentation: detectors — techniques: spectroscopic — telescopes

\section{INTRODUCTION}

SRAO (Seoul Radio Astronomy Observatory) has been operating $100 \mathrm{GHz}$ band receiver since its inauguration in 2002. It has suffered from poor angular resolution because the antenna diameter is only 6 meter. The Taeduk Radio Astronomy Observatory located nearby has been operating an array receiver at $100 \mathrm{GHz}$ band for its $14 \mathrm{~m}$ telescope. We believe both telescopes can benefit from similar angular resolution and work collaboratively, if the SRAO operates a $230 \mathrm{GHz}$ radio receiver. During the past 10 years, receiver technologies have been developed extensively for the ALMA (Atacama Large Millimeter Array) project, influencing local millimeter-wave radio astronomy community. One of the key receiver technologies is the phasing-type sideband separation mixer implemented with waveguide components taking smaller volume in a receiver cryostat. Moreover, this waveguide configuration easily allows image band to be terminated to a RF load at $4.2 \mathrm{~K}$ reducing noise further. In millimeter-wave range as noise contribution from a receiver becomes less dominant, a factor which mostly sets the sensitivity of a receiving system is the atmospheric transmission at a site. From the beginning phase of the $230 \mathrm{GHz}$ receiver development, one of the major concerns was regarding the atmospheric condition of the SRAO site. Simulations of zenith opacity show that when the air is dry, the opacity at $230 \mathrm{GHz}$ band can be lower than that at $115 \mathrm{GHz}$ (Holdaway \& Pardo 1997). Therefore it

Corresponding Author: J.-W. Lee is expected that the $230 \mathrm{GHz}$ band observations can be done sensitively at least for certain period in Korean dry winter. The operation limit of SRAO also depends on antenna surface accuracy. The SRAO 6 $\mathrm{m}$ antenna is known to have a surface accuracy better than $60 \mu \mathrm{m}$ (Koo et al. 2003) allowing its operation up to $300 \mathrm{GHz}$.

In order to best use the limited time slot, dual polarization mode is preferred (an array receiver may be a next step). In addition, we also wanted to develop wide bandwidth backend in order to provide good sensitivity in continuum mode observation and possibly to support spectrum survey observation.

We describe the frontend including our design concepts and requirements in Section 2, and backend system in Section 3. Control and observation software is described in Section 4. Test observations including the measurement of efficiencies are explained in Section 5.

\section{NEW FRONTEND}

\subsection{Design Cencepts}

The achievable receiver noise of a millimeter-wave receiver employing an SIS (Superconductor-InsulatorSuperconductor) mixer gets lowered to almost 2-3 times the quantum limit $h \nu / k \sim 12 \mathrm{~K}$ and hence the sky noise from the image sideband usually exceeds the double sideband (DSB) receiver noise. Thus the sky noise in the image sideband becomes a dominating factor of the system sensitivity in any spectrum observation (Jewell \& Mangum 1997). Therefore to keep 


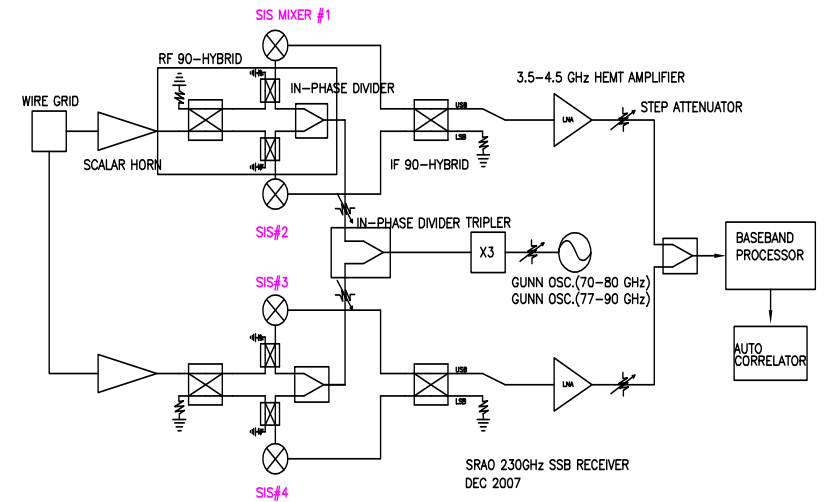

Fig. 1. - Schematic Diagram of the frontend of the 230 $\mathrm{GHz}$ receiver

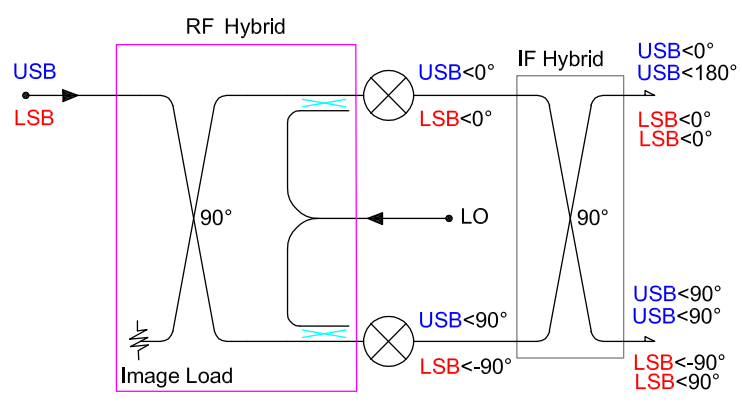

Fig. 2.- Principle of the phasing type sideband rejection

sensitivity in a system for spectrum observations with an SIS mixer receiver, employing sideband rejection is a natural choice.

Considering these backgrounds, several working groups have been successful in fabricating the hardware for the phasing type sideband rejection. The hardware is more compact and of lower loss than their quasi-optical version and even requires no mechanical adjustment. In order to increase the days of $230 \mathrm{GHz}$ operation per year we chose to add dual-polarization capability to the frontend. Based on these backgrounds, sideband rejection with orthogonal polarizations effectively to double integration time was adopted for the frontend configuration of the new receiver. The principal hardware concept, specifically sideband rejection (or separation if a system utilizes both sidebands) adopted in this project becomes practical owing mainly to the extensive, long-time engineered ALMA frontend development. The main goal in implementing rejection has been to adapt microwave frequency scheme of classic image rejection mixer to millimeter and submillimeter frequencies.

Fig. 1 shows the diagram of the frontend of the 230 $\mathrm{GHz}$ receiver. Polarization separation is done by a wire

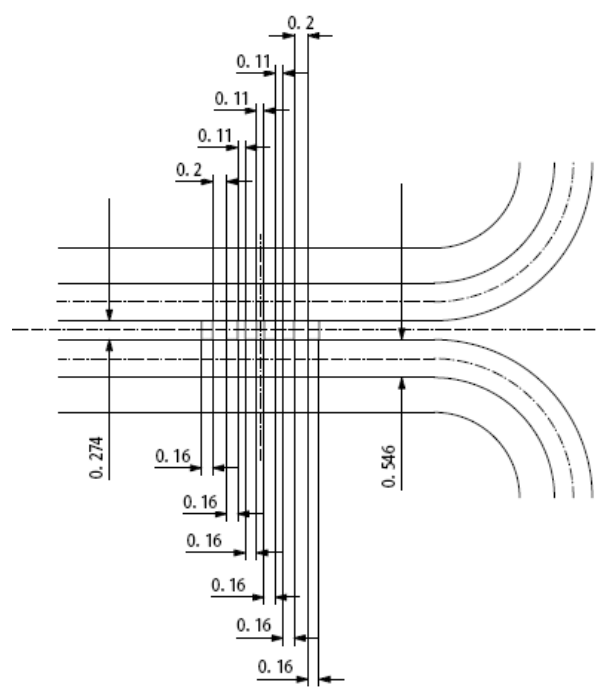

Fig. 3.- Dimensions of the RF quadrature hybrid for $210-270 \mathrm{GHz}$ (units in millimeters): top view. Excerpted from a drawing by Asayama (2004).

grid located in front of two feed horns. The weak point of this approach is to the introduction of an alignment problem between the grid and two feed horns when compared to the separation by an orthomode transducer. For each polarization two mixer chips biased separately take part and work as a single sideband (SSB) mixer. In total four mixer chips are needed to get dual-polarization capability. The RF quadrature hybrid giving $90^{\circ}$ phase delay to input, the vital component for sideband rejection, is located between the feed horn and the two mixers. The local oscillator signal generated from a WR-3.4 $(\times 3)$ frequency tripler is first split for each polarization from outside the dewar by another $3 \mathrm{~dB}$ hybrid, further divided half by $\mathrm{Y}$ divider, and eventually coupled to each mixer chip by a 15 $\mathrm{dB}$ coupler. Power attenuators are positioned after the warm divider and used for separate LO power pumping to each polarization. In principle all the sidebands can be separated (two IF outputs possible per each polarization) for further processing at IF stage, but in our case only upper sideband (USB) per polarization is amplified by a cryogenic preamplifier and gets further processed with the corresponding lower sideband (LSB) terminated by a $50 \Omega$ load.

\subsection{Sideband Rejection Implemented in Waveg- uide}

Sideband rejection in a mixer has been implemented in several ways. Passive filtering directly in front of a mixer or tuning a mixer reactively by a backshort can be categorized as direct methods but these methods intrinsically cause higher noise. Quasi-optical (QO) rejection scheme is relatively easier to fabricate than other methods and of low loss but tends to be bulky even in millimeter wavelengths. Most of the QO scheme 


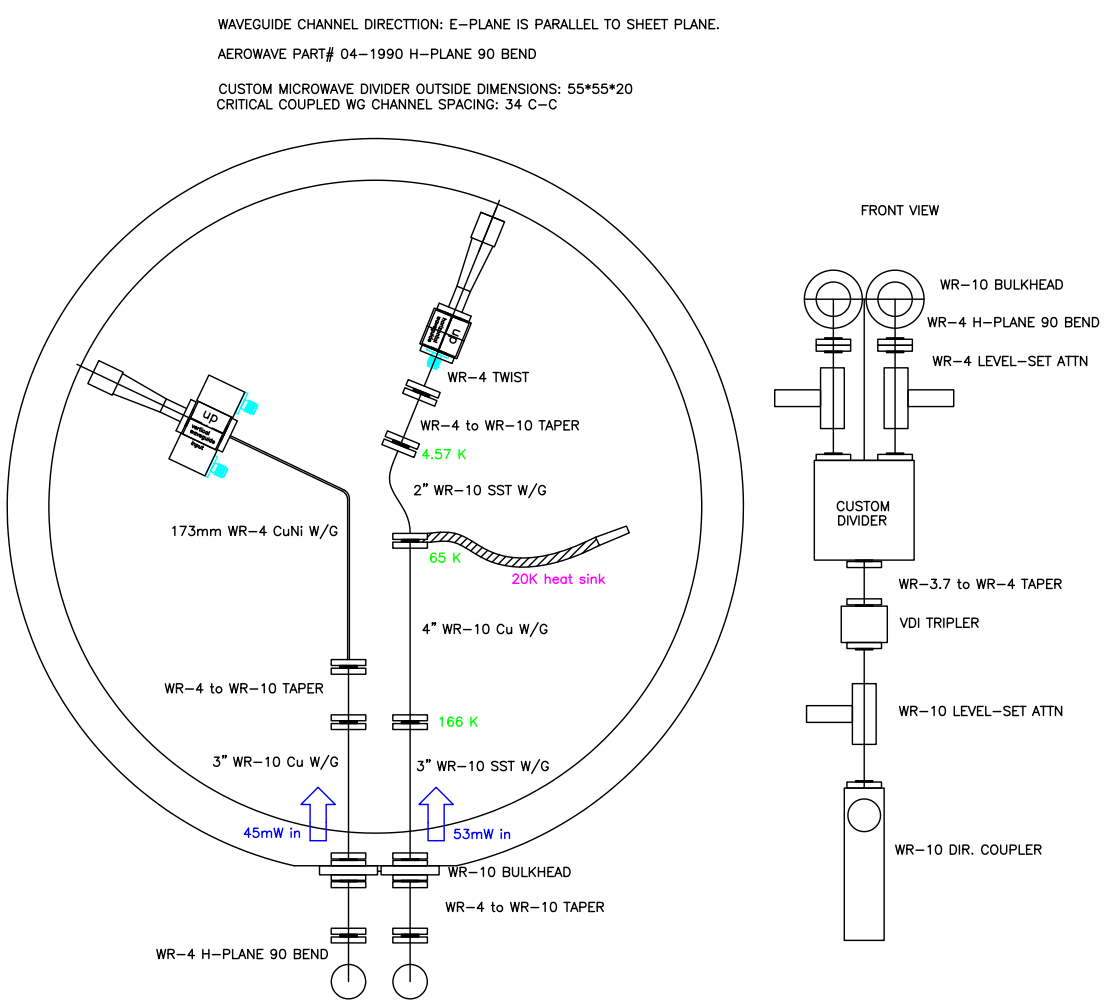

Fig. 4. - Waveguide run to a mixer of each polarization: top view, for overmoded operation, taper waveguides are located at both ends of WR-10 stainless steel waveguides. The warm LO circuit outside the dewar is shown on the right side under title "FRONT VIEW". Estimated physical temperatures along the right waveguide run are shown.

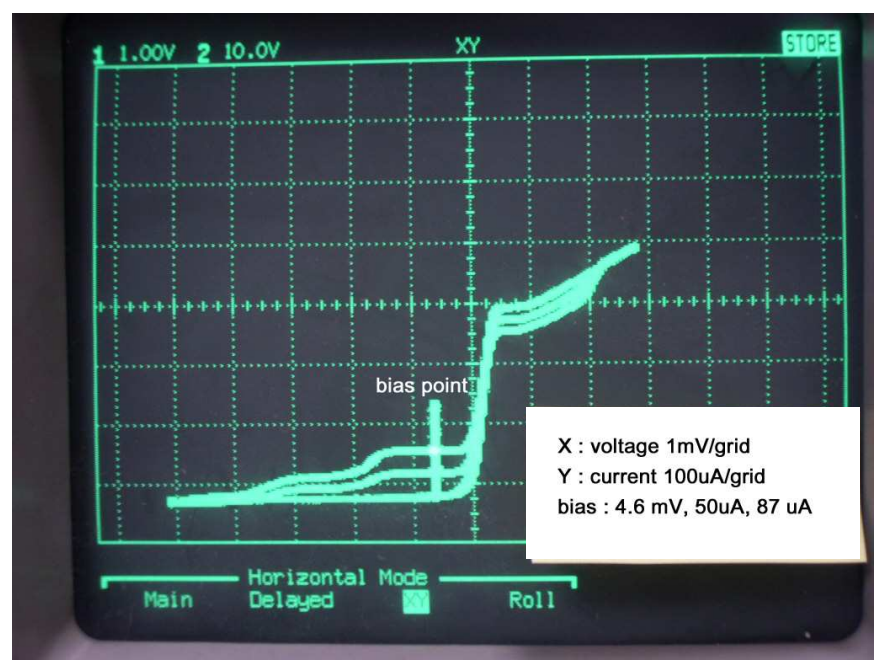

Fig. 5. - Capture of an oscilloscope CRT display showing pumped IV curves of the mixer F. The X, Y scales and bias point are as indicated. needs mechanical adjustment due to dependence of beam propagation on operating frequencies that operational fractional bandwidth is limited to $\leq 0.01$ without adjustment.

Finally waveguide implementation shown in Fig. 2, commonly brings manufacturing difficulties due to required structure sizes of $\sim \lambda / 10$ but this drawback is considered minor since the waveguide components are relatively compact in size and easily give wider fractional bandwidth of $\geq 0.25$ from conventional broadband matching technique (hence no tuning is needed for $\sim 60 \mathrm{GHz}$ instantaneous bandwidth at $230 \mathrm{GHz}$ ). Moreover when employed with SIS mixers the whole waveguide components can be cooled to a cryogenic temperature. Gold-plated waveguide components show almost 3 times less conductor loss at $4 \mathrm{~K}$ (Fingers \& Kerr 2008). Usually any waveguide termination is also cooled to have lower effective noise temperature.

The principle of the rejection lies in the property that when an RF signal is down-converted by a mixer each sideband is converted to have phase of different polarity. Therefore, if phase shift is given $90^{\circ}$ by an RF quadrature hybrid (functioning as a power divider with $90^{\circ}$ phase difference) then USB/LSB phases of 


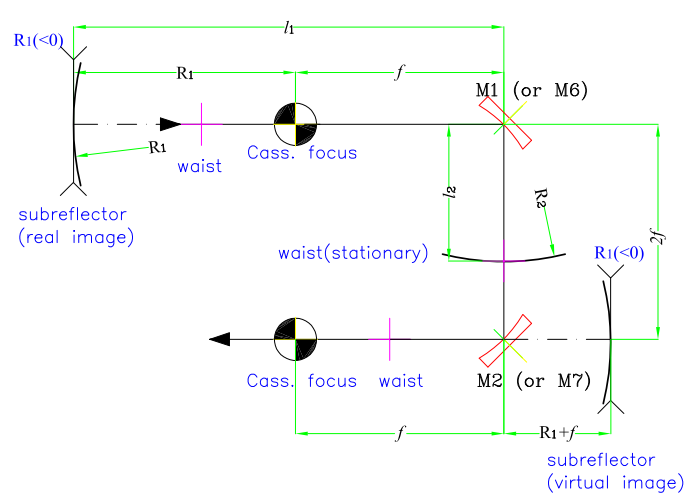

Fig. 6. - Imaging subreflector field to a location displaced by $2 f$ from the nominal Cassegrain focus, where $f$ is the focal length of M1 and M2. M1 and M2 have the same focal lengths and mirrored surface shapes; in effect the original Cassegrain focus field is replicated at $2 f$ to the bottom direction on this page. Only two identical mirrors are needed for any displacement.

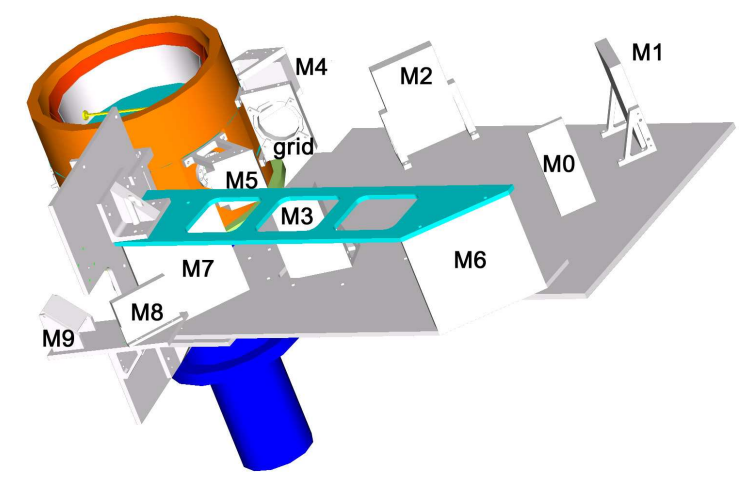

Fig. 7.- A perspective illustration of the whole receiver optics. M1 to M5 belong to the $230 \mathrm{GHz}$ optics and M6 to M9 are the $100 \mathrm{GHz}$ optics. M0 is a flat mirror near the Cassegrain focus which directs the telescope beam to either the $230 \mathrm{GHz}$ or the $100 \mathrm{GHz}$ optics.

the divided signal after conversion to IF frequency are $+0^{\circ},-0^{\circ}$ for the upper mixer and $+90^{\circ},-90^{\circ}$ for the lower mixer as shown in Fig. 2. The following power combining with $90^{\circ}$ phase shift by a 4 -port IF hybrid results in cancellation of the USB signal at the upper branch of the IF hybrid and cancellation of the LSB signal at the lower branch. This achieves, in effect, sideband separation. Note there is a termination load at the other input port of the RF hybrid. The sideband contrbutions from this load also experience the same phase polarity as that from the signal port but in this time USB/LSB separation is exchanged after the

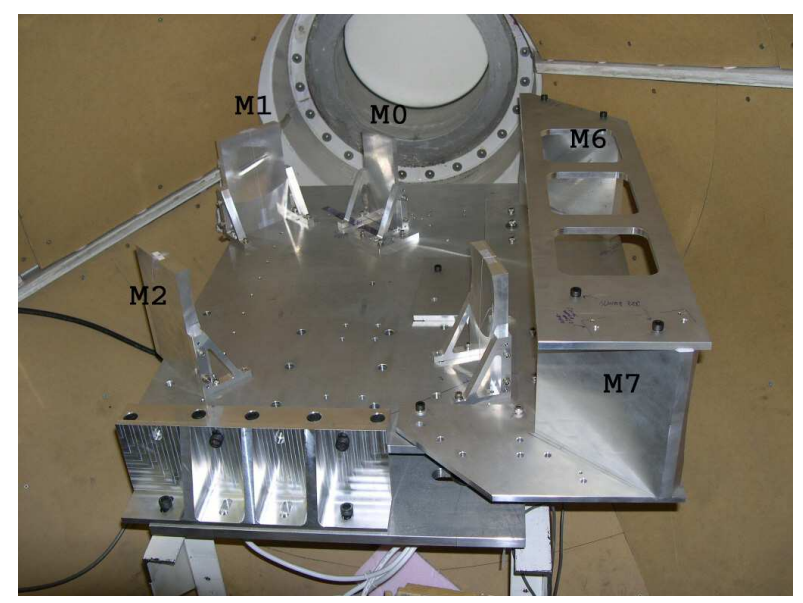

Fig. 8.- Optical components installed inside the receiver cabin. The vertex window of the telescope is seen at the top in this image.

IF hybrid. Therefore to keep system noise low the termination load should be at cold temperature. Exactly the same process happens during the sideband separation in an interferometer except that there is no RF hybrid but LO phase switching gives $90^{\circ}$ phase shift to the RF signal. Then a following complex correlator works as an IF hybrid. However, since atmospheric noise in the image band from each antenna is incoherent, there is no significant sensitivity enhancement by separation in an interferometer.

For the new receiver an RF hybrid and two $15 \mathrm{~dB}$ LO couplers connected to an Y divider were machined in a cube-shaped block. We adopted the block design by National Astronomical Observatory of Japan (Andoh et al. 2003), which was also used by other receiver developments (Nakajima et al. 2007). This RF hybrid has a typical form of a multi-section branch-line coupler and the most critical task involved was to get coupling branches machined within allowed tolerances. Fig. 3 shows the required dimensions of the coupler in the $\mathrm{RF}$ hybrid block with tolerances of $\pm 10 \mu \mathrm{m}$. Channel width connecting two main waveguides (WR-4) is $0.16 \mathrm{~mm}$ and the spacing of each channel is symmetrical with respect to the center line having two different spacing of $0.2 \mathrm{~mm}$ at the edge. In principle, the hybrid works for broader frequency range with more channels. Channel length of $0.274 \mathrm{~mm}$ is about $\lambda_{g} / 4$, where $\lambda_{g}$ is the guided wavelength.

\subsection{LO Circuit}

In general, to pump a millimeter-wave mixer LO signal can be injected in several ways: using a quasioptical diplexer, a slanted thin dielectric film, or conventional waveguide coupler. Using a diplexer usually produces mechanical complexity. Coupling by a thin dielectric film wastes much of LO power to reduce add- 

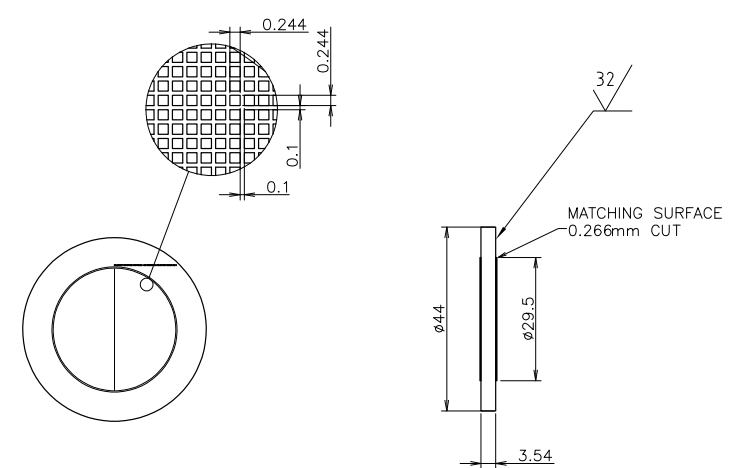

Fig. 9.- Dimensions of the vacuum window is shown where units are in millimeters. $0.244 \times 0.244$ region is the ridge and $0.1 \mathrm{~mm}$-wide stripes represent the machined-out valleys.

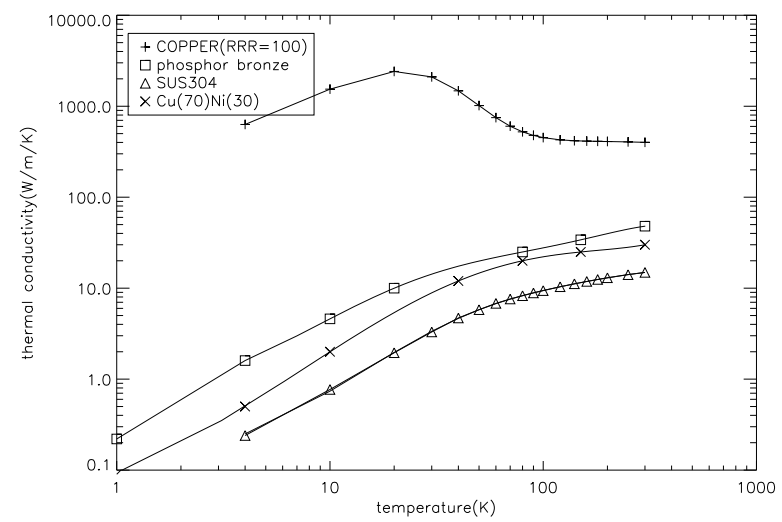

Fig. 10. - Thermal conductivity of the materials often used for the cryogenic heat transfer: from these curves thermal conductivity integrals are easily calculated. For example conductivity integral from $300 \mathrm{~K}$ to $20 \mathrm{~K}$ gives SST (20 $\mathrm{K}-300 \mathrm{~K})=3058 \mathrm{~W} / \mathrm{m}$ and $\mathrm{CuNi}(20 \mathrm{~K}-300 \mathrm{~K})=6754 \mathrm{~W} / \mathrm{m}$, thus using copper-nickel to connect $300 \mathrm{~K}$ with $20 \mathrm{~K}$ gives the $20 \mathrm{~K}$ stage twice bigger heat load than using stainless steel.

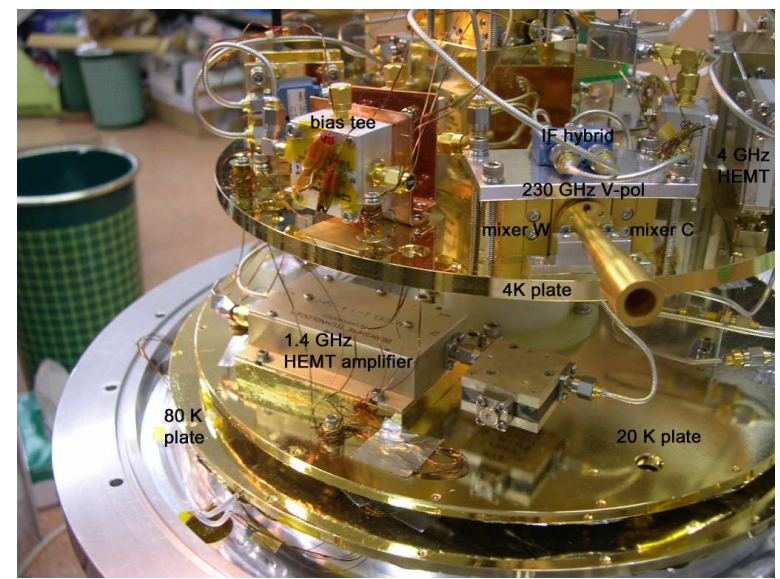

Fig. 11. - Inside layout of the receiver cryostat. There are $4 \mathrm{~K}, 20 \mathrm{~K}$ and $80 \mathrm{~K}$ thermal stages. The $20 \mathrm{~K}$ and $80 \mathrm{~K}$ stages have cylindrical shields wrapped with layers of superinsulation (removed for clarity). All the mixers are mounted on the $4 \mathrm{~K}$ plate.

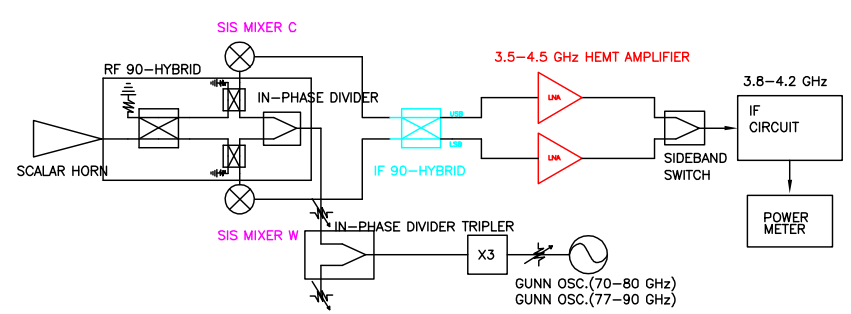

Fig. 12.- Measurement setup for SSB (2SB) noise temperature. A power meter measures output powers of the receiver for HOT and COLD loads in $400 \mathrm{MHz}$ bandwidth.

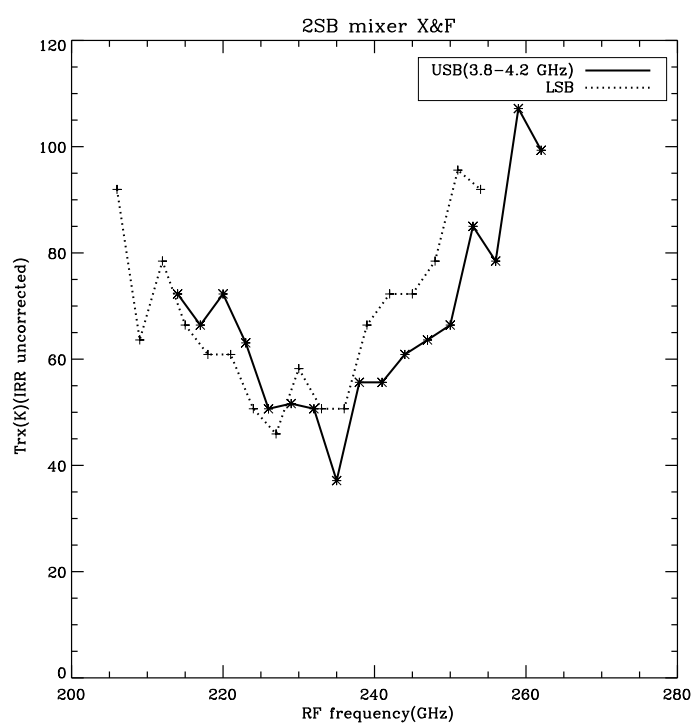

Fig. 13. - Uncorrected $T_{R X, D S B}$ of the mixer pair $X$ and F.

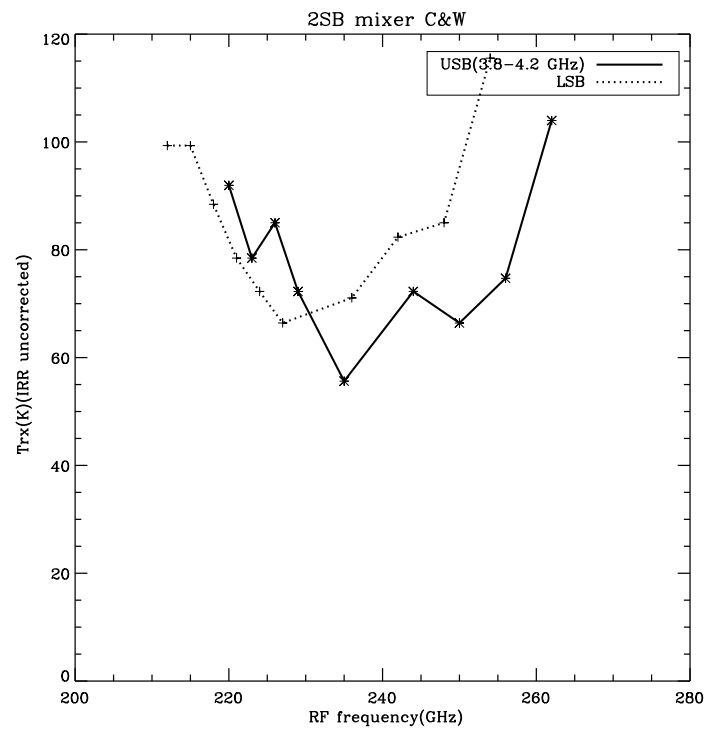

Fig. 14. - Uncorrected $\mathrm{T}_{\mathrm{RX}, \mathrm{DSB}}$ of the mixer pair $\mathrm{C}$ and W 
Table 1.

Loss estimate of the waveguide run

\begin{tabular}{lc}
\hline \hline component & loss $(\mathrm{dB})$ \\
\hline 5" WR-10 SST WG, V-pol & 3.3 \\
173 mm WR-4 CuNi ${ }^{\mathrm{a}} \mathrm{WG}, \mathrm{H}-\mathrm{pol}$ & 8.6 \\
tapered transition & \\
(both WR-4 to WR-10) & 1 \\
LO Y divider & $3^{\mathrm{b}}$ \\
LO coupler to SIS chips & $15^{\mathrm{b}}$ \\
\hline V-pol (total) & 22.3 \\
H-pol (total) & 26.6 \\
\hline Gunn oscillator output & $30 \mathrm{~mW}$ \\
tripler efficiency & $3 \%$ \\
output from tripler & $0.9 \mathrm{~mW}(\sim 0 \mathrm{dBm})$ \\
\hline
\end{tabular}

(a) loss: $0.5 \mathrm{~dB} / \mathrm{cm}$ in manufacturer's data, 0.66 $\mathrm{dB} / \mathrm{cm}$ at $230 \mathrm{GHz}$ when measured with the Erickson power meter (b) coupling loss, not insertion loss

ed noise. In addition, both quasi-optical methods need separate horns for transmitting LO power. For the new receiver, we chose a waveguide coupler with a divider which is most compatible with the RF hybrid.

LO driving using the fundamental-mode waveguides has been avoided since the loss of cryogenic waveguides gets exceedingly high with increasing frequency. At 230 $\mathrm{GHz}$ standard WR-4 waveguide has loss of $117 \mathrm{~dB} / \mathrm{m}$ for a stainless steel (SST) waveguide while $18 \mathrm{~dB} / \mathrm{m}$ for a normal copper waveguide. Instead WR-10 waveguide has loss of $26 \mathrm{~dB} / \mathrm{m}$ for an SST waveguide and $3.8 \mathrm{~dB} / \mathrm{m}$ for a copper waveguide (Ediss 2003). Overmoded (oversized) transmission of the LO signal has received attention to alleviate this high loss.

The frequency range of the fundamental-mode operation of WR-10 waveguide is 75 to $110 \mathrm{GHz}$ and even signal frequency higher than $110 \mathrm{GHz}$ can be guided in an overmoded operation using the same waveguide. However, in this overmoded operation, the fundamental mode can couple into one of the other propagating modes. The coupling to the other modes should be avoided and that can be guaranteed if there is no abrupt discontinuity through oversized waveguide run. Thus if a bending waveguide is required along a waveguide run, a bending waveguide should be used in fundamental-mode operation, not in overmoded operation. In our case, only straight WR-10 waveguide sections are used in overmoded operation.

The LO circuit is shown in Fig. 4. Estimated conductive heat load along each waveguide run is $\sim 45 \mathrm{~mW}$ and $\sim 53 \mathrm{~mW}$, respectively for each polarization. Also what needs to be checked in an LO circuit is whether delivered power is ample to drive a parallel-coupled twin junction (PCTJ) SIS mixer. Typical pumping power for a PCTJ SIS mixer is $P_{L O} \sim 8(h \nu / e)^{2} / R_{N} \sim$ $10^{-5}-10^{-4} \mathrm{~mW}$ for $R_{N} \sim 20 \Omega$. Estimated loss of the waveguide runs given in Table 1 and output power assumed $\sim 0 \mathrm{dBm}$ from the tripler guarantee that the delivered power of the LO circuit is at least 10 times greater than needed. Fig. 5 shows an oscilloscope capture of the pumped IV curves of the mixer F. All SIS mixer chips in the receiver were fabricated and provided by NAOJ (Asayama 2004).

\subsection{Receiver Optics}

Due to shape of the new dewar cryostat and the location of feed horns on $4 \mathrm{~K}$ plate inside the cryostat, the telescope beam needs to be relayed farther from the nominal Cassegrain focus of the $6 \mathrm{~m}$ telescope. We utilized a simple relation of a fundamental Gaussian beam: if the first mirror (say M1) is located at the distance equal to its focal length $f$ away from the nominal geometrical focus of a given Cassegrain telescope, an image of the subreflector is formed on the opposite side, $l_{2}$, from the mirror and the image is a waist $\left(R_{2}\right.$, the radius of phase curvature of image becomes infinite) as shown in Fig. 6. If the second mirror (say M2) is positioned at double the focal length $2 f$ from the first mirror, the beam between the first and the second mirror is exactly symmetrical since the image (it should be stationary) at $l_{2}$ is the waist. Effectively the input/output beams of $\mathrm{M} 1$ are replicated at M2 again with mirror symmetry about $l_{2}$. The optics for the legacy $100 \mathrm{GHz}$ mixer has been also designed in this way (M6 and M7, see Figs. 7 and 8). Two additional mirrors that follow relay the beam at M2 to each feed horn in frequency-independent manner (M3-M4, M3-M5). These optical components were aligned with a laser in lab before final installation in the telescope cabin.

The vacuum window is a flat plastic plate. Motheye pattern was machined on both surfaces for antireflection matching layers (see Fig. 9). This pattern is intrinsically polarization-insensitive when compared with concentric grooved pattern. To avoid grating effect, pitch to wavelength ratio should be made small. In our case, the ratio is 0.27 at $235 \mathrm{GHz}$ and the valley (machined-out part) width is set to $0.1 \mathrm{~mm}$. The depth is the usual $\lambda /\left(4 n_{e f f}\right) \sim 0.27 \mathrm{~mm}$ where $n_{\text {eff }}=1.2$, the effective refractive index between air and the window material, Teflon. The thickness of the window is 3.54 $\mathrm{mm}$. With this thickness estimated insertion loss is $\leq$ $0.1 \mathrm{~dB}$. Estimated flexure at the center of the window under the pressure of $1 \mathrm{~atm}$ is about $\leq 0.5 \mathrm{~mm}$ in the worst case.

\subsection{Cryogenics}

With a number of components installed on top of the $4 \mathrm{~K}$ plate and a large chamber, cryogenic thermal design is also critical for optimal operation. The radiative 


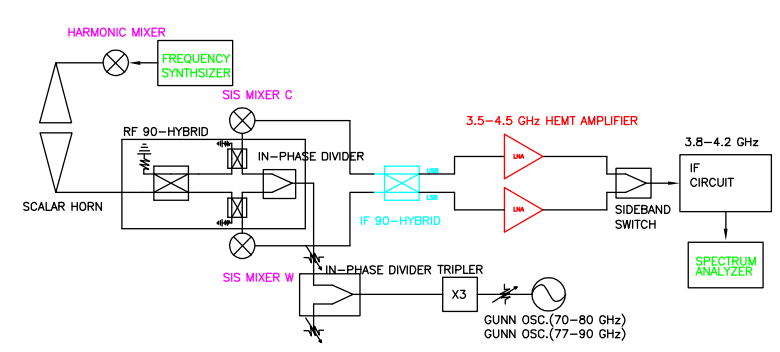

Fig. 15. - Measurement setup for sideband rejection

heat load to the $80 \mathrm{~K}$ stage from $300 \mathrm{~K}$ is approximately $225 \mathrm{~W}$. Even one layer of superinsulation wrapped on the $80 \mathrm{~K}$ stage is known to drop the heat load to 10 W. Additional wrapping does not provide a direct linear increase in efficiency due to effect of conductive transfer in proximity. Ten layers of superinsulation are expected to drop radiative load by $1 / 100$ according to an experimental data (Kooi 2001). The load from the $80 \mathrm{~K}$ shield to the $4 \mathrm{~K}$ stage is estimated conservatively to be about $1.3 \mathrm{~W}$. Since all the RF components are located on top of the $4 \mathrm{~K}$ plate, the actual surface area is larger and accordingly the radiative load can be higher than this value. Therefore we adopted additional shield to the $20 \mathrm{~K}$ stage. Then the load from the $20 \mathrm{~K}$ shield to the $4 \mathrm{~K}$ stage can be reduced to $\sim 5 \mathrm{~mW}$. From the $80 \mathrm{~K}$ shield to the $20 \mathrm{~K}$ shield, the radiative load is about $0.92 \mathrm{~W}$ without insulation. This load to the 20 $\mathrm{K}$ stage is still substantial for optimal $4 \mathrm{~K}$ operation so several layers of superinsulation were wrapped to the $20 \mathrm{~K}$ shield during assembly.

Conductive heat load was estimated by fitting the thermal conductivity $\mathrm{K}(\mathrm{T})$ in a polynomial form, and later integration was done to get thermal conductivity integral, $\int_{\mathrm{T}_{1}}^{\mathrm{T}_{2}} \mathrm{~K}(\mathrm{~T}) \mathrm{dT}$. Fig. 10 shows compiled conductivity curves for the materials used for this receiver (Simon \& Drexler 1992). These data were also used to arrive at the temperature distribution along the waveguide run in Fig. 4. Fig. 11 shows the internal thermal stages of the receiver cryostat with radiation shields removed.

\subsection{Frontend Performances}

The receiver noise temperatures were measured with the setup shown in Fig. 12. Both sidebands of each polarization were connected to two cryogenic HEMT amplifiers and standard Y-factor measurements were done using a microwave power meter. Optimum bias point of each mixer was searched around the first photon step and determined experimentally. It was found that over the RF frequency range when the mixer pair is biased at the same voltage the receiver noise shows its minimum. The current of each mixer under a fixed voltage bias is not controllable since the current flowing in each mixer of the pair depends on degree of matching of the LO signal to each mixer. If two mixers are assumed

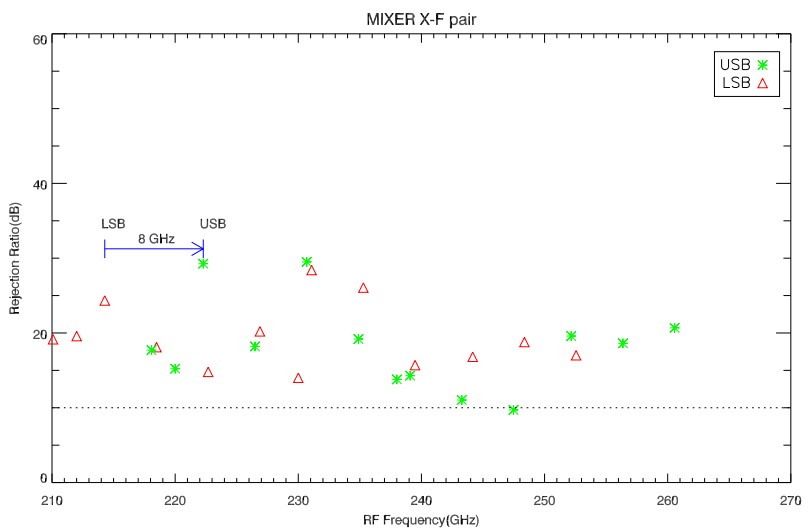

Fig. 16. - Sideband rejection ratio for the mixer pair $\mathrm{X}$ and F. With fixed LO frequency of $218 \mathrm{GHz}$ two rejection ratios at the RF frequency of $214 \mathrm{GHz}$ (LSB) and at the RF frequency of $222 \mathrm{GHz}$ (USB) were obtained and the same to other LO frequencies.

to have identical DSB noise temperature of $\mathrm{T}_{\text {mix,DSB }}$ and sideband ratio of each mixer is ideally unity then attainable SSB noise temperature is $2 \mathrm{~T}_{\text {mix,DSB }}+\mathrm{T}_{\text {load }}$ where $T_{\text {load }}$ is the noise temperature of the termination load in the RF hybrid block. Measurement results are shown in Figs. 13 and 14. TRX,DSB denotes the noise temperature of one sideband obtained from the standard Y-factor method which usually defines actual DSB receiver noise temperature of a DSB receiver assuming equal sideband ratio. Since an SSB mixer has not ideal rejection unequal sideband ratio has to be introduced in deducing correct SSB noise when broadband hot and cold loads are used. Hence corrected receiver noise is given by $\mathrm{T}_{\mathrm{RX}, 2 \mathrm{SB}}=(1+1 / \mathrm{R}) \mathrm{T}_{\mathrm{RX} \text {,DSB }}$ where $\mathrm{R}$ is the image rejection ratio. Since the measured image rejection ratio is always greater than $10 \mathrm{~dB}$, approximate correction factor to get $\mathrm{T}_{\mathrm{RX}, 2 \mathrm{SB}}$ is 1.1. The measured noise temperatures are below $100 \mathrm{~K}$ for $210-260 \mathrm{GHz}$ $\mathrm{RF}$ frequency band.

The setup for the sideband rejection is different from the noise measurement setup as shown in Fig. 15. Since at millimeter-wave band knowing absolute RF signal level is too difficult, relative measurements to remove an unknown absolute amplitude are needed(Kerr et al. 2001). The measurements are listed as follows.

(i) $\mathrm{CW}(\mathrm{USB})$ to IF(USB) and CW(USB) to IF(LSB) response

(ii) $\mathrm{CW}(\mathrm{LSB})$ to $\mathrm{IF}(\mathrm{USB})$ and $\mathrm{CW}(\mathrm{LSB})$ to $\mathrm{IF}(\mathrm{LSB})$ response

(iii) $\mathrm{RF}$ (HOT) to IF(USB) and $\mathrm{RF}(\mathrm{COLD})$ to $\mathrm{IF}(\mathrm{USB})$ response

(iv) $\mathrm{RF}(\mathrm{HOT}$ ) to $\mathrm{IF}(\mathrm{LSB})$ and $\mathrm{RF}(\mathrm{COLD})$ to $\mathrm{IF}(\mathrm{LSB})$ response

CW means an RF source in the corresponding RF frequency and RF (HOT) is a measurement using a microwave absorber at room temperature. RF (COLD) is a measurement with an absorber in liquid nitro- 


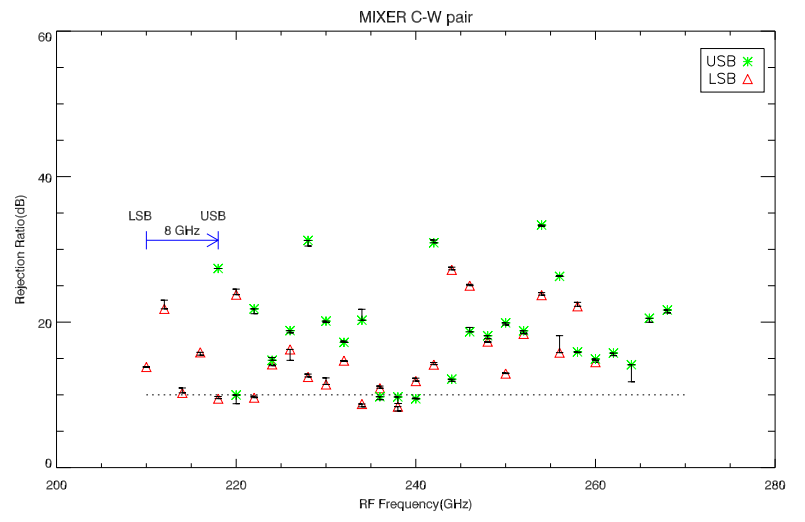

Fig. 17. - Sideband rejection ratio for the mixer pair $\mathrm{C}$ and $\mathrm{W}$

gen. The CW at $210-260 \mathrm{GHz}$ was generated using a frequency synthesizer connected to a WR-4 harmonic mixer, and the CW signal was transmitted by a standard gain feed horn. All the measurements were done with a spectrum analyzer. The derived image rejection ratios over the whole RF frequency are shown in Figs. 16 and 17. As noted, the rejection ratio is better than $10 \mathrm{~dB}$ at $210-260 \mathrm{GHz}$ band.

\section{IF CIRCUITS AND SPECTROMETERS}

We have replaced the old narrow band $(100 \mathrm{MHz})$ autocorrelation spectrometer with two commercial FFT spectrometers with $1 \mathrm{GHz}$ bandwidths and $2^{14}$ channels. The IF signal from the frontend needs to be fed into the spectrometer via long cables. IF band just after dewar is $3.5-4.5 \mathrm{GHz}$, while the input frequency range of the FFT spectrometer is $0-1 \mathrm{GHz}$. Down conversion is done in two steps to use the legacy $0.9-1.9$ $\mathrm{GHz}$ IF processor as shown in Fig. 18. This double conversion also helps to avoid harmful radio interference by artificial signals. In the first down-conversion, lower sideband is selected using $5.4 \mathrm{GHz}$ LO to eliminate spurious signals at $2.6 \times 2-4.2 \mathrm{GHz}=1 \mathrm{GHz}$ or $2.6 \times 2-3.3 \mathrm{GHz}=1.9 \mathrm{GHz}$ that would occur if $\mathrm{LO}$ frequency would be chosen as $2.6 \mathrm{GHz}$. After the first down-conversion, an $1.9 \mathrm{GHz}$ fixed oscillator is used to convert the $0.9-1.9 \mathrm{GHz}$ IF band to $\mathrm{DC}-1.0 \mathrm{GHz}$ base band. We have not made seperate circuit for continuum power measurement, contrary to the previous one (Koo et al. 2003). Since the FFT spectrometer covers full IF band, we simply use the spectrometer for the measurement. Dynamic range of the spectrometer is rather high as 8 bits in voltage or $48 \mathrm{~dB}$ in power.

\section{SOFTWARE}

Backend system uses two FFT spectrometers for dual polarization observation. The spectrometer (Benz et al. 2005) largely consists of analog-to-digital converter (ADC) and Field-Programmable Gate Array

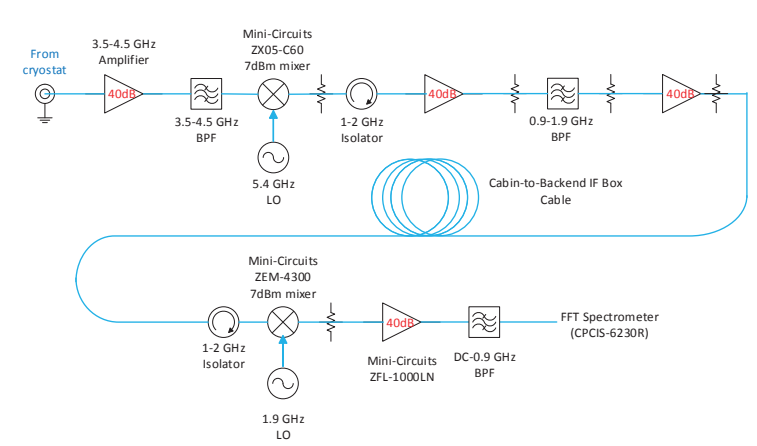

Fig. 18. - IF circuit in the antenna cabin and the baseband circuit in front of the backend spectrometer are shown for each polarization. The label attached to each component is self-explanatory with the corresponding model number shown for commercially available products.

(FPGA). The ADC can process data with 8 bits resolution at $2 \mathrm{Gs} / \mathrm{s}$ sample rate. The data are transferred to FGPA at a speed of $62.5 \mathrm{MHz}$. One data set has 32,768 samples and all subsequent processing is conducted in the FPGA unit. Therefore a spectrum consists of 16,384 or $2^{14}$ channels. During FFT calculation, two processors run alternately and data are accumulated in one buffer area. The other buffer is used for transfer of data to computer. It takes $16.384 \mu$ s to acquire one data set and over one thousand data sets can be accumulated. Allan time is found to be near 2000 s (Benz et al. 2005). Backend control system consists of one single board computer (SBC), where CPU is intel Atom D525 processor, and OS is Ubuntu 10.04 of kernel 2.6.33. The SBC controls two FFT spectrometers via compact PCI bus. Backend control program was compiled with GNU C compiler. Thread functions are used to minimize time delay in controlling two spectrometers. The program receives observation commands from an operator computer via TCP/IP socket (Byun \& Yun 2002). Socket communication generally induces a time delay between computers. The time delay can cause problem when LO frequency is out of lock or telescope pointing is in error, since integration must be halted promptly in these cases. To solve this problem, we provided direct link from the external I/O port of the spectrometer, to LO frequency monitor and telescope motor controller.

\section{TEST OBSERVATION AND EFFICIENCY MEASUREMENTS}

\subsection{Test Observation}

Development of the $230 \mathrm{GHz}$ frontend was completed on December 2007 and retrofitting the IF hardware and the adoptation of the state-of-art FFT spectrometers was finished on May 2013.

Several years of operation of $230 \mathrm{GHz}$ receiver system indicates that definitely for typical winter season 
Table 2.

Beam size and efficiencies

\begin{tabular}{ccccc}
\hline \hline $\begin{array}{c}\text { Frequency } \\
(\mathrm{GHz})\end{array}$ & $\begin{array}{c}\text { FWHM } \\
\left({ }^{\prime \prime}\right)\end{array}$ & $\begin{array}{c}\text { Aperture eff. } \\
(\%)\end{array}$ & $\begin{array}{c}\text { Beam eff. } \\
(\%)\end{array}$ & $\begin{array}{c}\text { Moon eff. } \\
(\%)\end{array}$ \\
\hline 219 & 50 & 59 & 61 & 80 \\
244 & 45 & 49 & 51 & 86 \\
266 & 41 & 44 & 47 & 82 \\
\hline
\end{tabular}

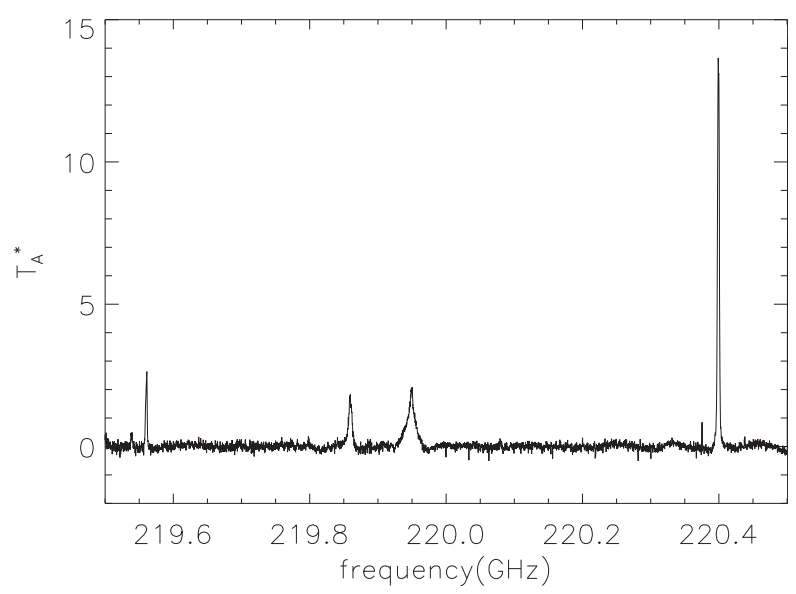

Fig. 19. - Spectrum taken with the FFT spectrometer with $1 \mathrm{GHz}$ bandwidth

from November to March, sky condition of southern Korean peninsula enables observation at $1.3 \mathrm{~mm}$ window. The system temperature during this time is found to be $200-400 \mathrm{~K}$ at $230 \mathrm{GHz}$ on clear days. We have conducted test observations toward many well known objects like Orion KL. Shown is the spectrum toward Orion KL obtained with the wide band FFT spectrometer in Fig. 19. On either sides, $\mathrm{C}^{13} \mathrm{O}(2-1)$ and $\mathrm{C}^{18} \mathrm{O}(2-1)$ spectra are seen. SO $65-5_{4}$ transition is found at $219.95 \mathrm{GHz}$. When compared with Sutton et al. (1985), several lines are missing, probably due to poor pointing and sensitivity at the time of observation. A line at $219.85 \mathrm{GHz}$ is not identifed in Sutton et al. (1985). We inspected the possibility of a feature by poor baseline or by penetration from the other side band, but these are not likely.

\subsection{Efficiency Measurements}

We made observations toward planets like Jupiter, Saturn, and Mars to measure beam size and apeture and beam efficiencies. For this, Gaussian beam and uniform disk model for planets are assumed (Koo et al. 2003). The brightness temperatures of the planets are taken from Mangum (1993). The measured efficiencis are listed in Table 2, which shows measured efficiencies are found to be consistent with those for $100 \mathrm{GHz}$ (Koo et al. 2003).

\section{CONCLUSIONS}

In order to open $1.3 \mathrm{~mm}$ window in Korea, we developed a dual polarization SIS receiver adopting waveguide sideband separation function. Receiver noise temperature is lower than $100 \mathrm{~K}$. IF processing units are retrofitted accordingly, and new wide band FFT spectrometers have been installed and the associated software devleoment was done. After mounting the system to the $6 \mathrm{~m}$ telescope, we obtained system temperatures as good as $150 \mathrm{~K}$ during winter time. By observing planets we measured aperture and beam efficiencies, typically $44-59 \%$ and $47-61 \%$, respectively in the frequency range of $210-260 \mathrm{GHz}$. We also conducted test observations of many standard objects. After test observations several observation programs have been carried out and a few of them have already been published (Lee et al. 2010, 2012). These observations verify the performance of the new $230 \mathrm{GHz}$ receiving system. It is concluded that at least for four months during winter, sky conditions are good enough to allow to open the $1.3 \mathrm{~mm}$ window.

\section{ACKNOWLEDGMENTS}

Authors thank support by Drs. Noguchi and Asayama at National Astronomical Observatory, Japan. Authors are greatful for the financial support for the purchase of the FFT spectrometers by Prof. Trippe at Seoul National University, Korea. This work has been partially supported by Korea Basic Science Institute grant.

\section{REFERENCES}

Andoh, H., Minamidani, T., Mizuno, N., Mizuno, A., Fukui, Y., Asayama, S., Yonekura, Y., \& Ogawa, H. 2003, Designs of Wideband 3 dB Branch-Line Couplers for ALMA Bands 3 to 10, ALMA Memo No. 468

Asayama, S. 2004, private commnunications

Benz, A. O., Grigis, P. C., Hungerbühler, V., Meyer, H., Monstein, C., Stuber, B., \& Zardet, D. 2005, A Broadband FFT Spectrometer for Radio and Millimeter Astronomy, A\&A, 442, 767 
Byun, D.-Y., \& Yun, Y.-Z. 2002, Development of Control Softwares for the SRAO 6-Meter Telescope Based on PCs Running Linux, Experimental Astronomy, 14, 183

Ediss, G. 2003, Measurements and Simulation of Overmoded Waveguide Components at $70-118 \mathrm{GHz}$, 220-330 GHz and 610-720 GHz, ALMA Memo No. 467

Fingers, R., \& Kerr, A. R. 2008, Microwave Loss Reduction in Cryogenically Cooled Conductors, Int. J. Infrared and Millimeters Waves, 29, 924

Jewell, P. R., \& Mangum, J. G. 1997, System Temperatures, Single Versus Double Sideband Operation, and Optimum Receiver Performance, ALMA Memo No. 170

Holdaway, M. A., \& Pardo, R. R. 1997, Modeling of the Submillimeter Opacity on Chajnantor, MMA Memo 187

Kerr, A. R., Pan, S.-K., \& Effland, J. E. 2001, Sideband Calibration of Millimeter-Wave Receivers, ALMA Memo No. 357

Koo, B. C., Park, Y.-S., Hong, S. S., Yun, H.-S., Lee, S.-G., Byun, D.-Y., Lee, J.-W., Choi, H.-K., Lee, S.-S., Yoon, Y.-Z., Kim, K.-T., Kang, H. W., \& Lee, J.-E. 2003, Performance of the SRAO 6-Meter Radio Telescope, JKAS, 36, 43

Kooi, J. W. 2001, http://www.submm.caltech.edu/cso/

Lee, J.-E. Lee, H.-G., Shinn, J.-H., Dunham, M. M., Kim, I.-S., Kim, C. H., Bourke, T. L., Evans, N. J., \& Choi, Y. 2010, The Spitzer c2d Survey of Nearby Dense Cores: Jet and Molecular Outflow Associated with a Young Stellar Object in Core A of L1251, ApJ, 709, 74

Lee, J.-W., Koo, B.-C., \& Lee, J.-E. 2012, CO J=2-1 Line Observations toward the Supernova Remnant G54.1+0.3, JKAS, 45, 117

Mangum, J. G. 1993, Main-Beam Efficiency Measurements of the Caltech Submillimeter Observatory, PASP, 105, 117

Nakajima, T., Kimura, T., Nishimura, A., Iwashita, H., Miyazawa, C., Sakai, T., Iono, D., Kohno, K., Kawabe, R., Kuno, N., Ogawa, H., Asayama, S., Tamura, T., \& Noguchi, T. 2007, A New 60 cm Radio Survey Telescope with the Sideband-Separating SIS Receiver for the $200 \mathrm{GHz}$ Band, PASJ, 59, 1005

Simon, N. J., \& Drexler, R. P. 1992, Properties of Copper and Copper Alloys at Cryogenic Temperatures, NIST monograph \#177, 7

Sutton, E. C., Black, G. A., Masson, C. R., \& Phillips, T. G. 1985, Molecular Line Survey of Orion A from 215 to $247 \mathrm{GHz}$, ApJS, 58, 341 